Disclosure of Interest: H. Deckx Employee of: Galapagos NV, Belgium, S Hatch Consultant for: Galapagos NV, Belgium, M. Robberechts Employee of: Galapagos NV, Belgium, S. Dupont Employee of: Galapagos SASU, France, J. Desrivot Employee of: has been employee of Galapagos SASU, France, H. Coleman Paid instructor for: Covance has been contracted by Galapagos NV to conduct the study, S. Larsson: None declared, A. Struglics: None declared, E. van der Aar Employee of: Galapagos NV, Belgium, A. Fiew Employee of: Galapagos NV, Belgium

DOI: 10.1136/annrheumdis-2018-eular.3101

\section{FRI0540 IDENTIFICATION OF BIOMARKERS OF OA ASSOCIATED TO DEFECTIVE AUTOPHAGY}

1. Lorenzo-Gomez ${ }^{1}$, N. Oreiro ${ }^{2}$, J.A. Pinto-Tasende ${ }^{2}$, F.J. Blanco ${ }^{1,2}$, B. Carames ${ }^{1}$. ${ }^{1}$ Rheumatology Division. Cartilage Biology Group, The Institute of Biomedical Research of A Coruña (INIBIC); ${ }^{2}$ Clinical Rheumatology Division, Complejo Hospitalario Universitario A Coruña (CHUAC), A coruña, Spain

Background: In osteoarthritis (OA), defects in cellular homeostasis, and in particular in autophagy, are evident and precede joint damage. In this sense, we have shown that there is a defect in autophagy in OA human chondrocytes and cartilage, and pharmacological activation of autophagy protects against joint damage. These data suggest that joint damage could be due, at least in part, to a failure of autophagy, by inducing an abnormal accumulation of cellular products related to disease.

Objectives: These observations represent a unique opportunity to identify and validate potential biomarkers associated with autophagy defects that could facilitate the development of therapeutic strategies to prevent OA progression.

Methods: A comparative analysis of 86 autophagy genes was performed in blood from non-OA and knee OA patients. Non-OA patients (Age: $61,17 \pm 1,370$ years; BMI: 25,76 $\pm 0,69$; Sex: Females; $n=12$ ) and Knee OA patients (Age: $65,75 \pm 1,528$ years; BMI: $30,25 \pm 0,88$; Sex: Females; $n=12$, OA grade III-IV) were profiled using a human autophagy PCR array (PrimePCR autophagy human panel, BioRad) and analysed using the PrimePCR analysis software, Biorad. In addition, we performed a quantitative proteomic analysis of defective autophagy by genetic deletion of Atg5 in human OA chondrocytes by using ITRAQ (isobaric tags for relative and absolute quantitation) labelling coupled with on-line 2D LC/MS/MS. Protein identification and quantification were performed using Protein Pilot Software 4.0. Each MS/MS spectrum was searched in the Uniprot/Swissprot database for Homo sapiens.

Results: 16 autophay-related genes were significantly down-regulated in blood from knee OA patients compared to non-OA patients. No significant up-regulation was observed in blood from Knee OA patients, however a trend-toward up-regulation was detected in several genes involved in the mTOR signalling pathway. Importanly, 5 key autophagy-related genes, such as, ATG16L2, ATG12, ATG7, ATG4B and MAP1LC3B involved in initiating autophagy, phagophore extension and autophagosome formation were significant downregulated in knee OA patients compared to non-OA patients $(p<0.05)$. Interestingly, HSP90AA1 and HSPA8, a chaperone-mediated autophagy genes involved in stress response and protein folding, were significant downregulated $(p<0.001)$ in blood from knee OA patients. In addition, several regulators of autophagy and apoptosis, such as BNIP3, BCL-2 and BCL2L1 were a significantly downregulated in OA patients $(p<0.01)$. Total proteome screening in human OA chondrocytes with defective autophagy, showed a significant reduction of Heat shock protein HSP90-alpha (HSP90A1) $(p<0.05)$, suggesting that reduced autophagy is associated to OA pathology and could be a potential biomarker for $O A$ progression and development.

Conclusions: This approach represents an unique opportunity to identify and validate early-stage biomarkers associated with defective autophagy that could facilitate the development of therapeutic strategies to prevent joint damage.

Disclosure of Interest: None declared

DOI: 10.1136/annrheumdis-2018-eular.5527

\section{FRI0541 INCREASING A PERSON'S OWN PHYSICAL ACTIVITY AND STRENGTH CAN MINIMISE CARTILAGE VOLUME LOSS IN OLDER-ADULTS: A BETWEEN- AND WITHIN- PERSON ANALYSIS ON A POPULATION-BASED PROSPECTIVE COHORT}

I.P. Munugoda ${ }^{1}$, S. Balogun ${ }^{1}$, K. Wills ${ }^{1}$, F. Cicuttini ${ }^{2}$, G. Jones ${ }^{1}$, M.L. Callisaya ${ }^{1}$, D. Aitken ${ }^{1} .{ }^{1}$ Menzies Institute for Medical Research, University of Tasmania, Hobart, Tasmania; ${ }^{2}$ Department of Epidemiology and Preventive Medicine, Monash University Medical School, Melbourne, Victoria, Australia

Background: The relationship between physical activity (PA) and osteoarthritis (OA) has been controversial, with some studies showing a detrimental effect and others showing either no effect or a beneficial effect. Traditionally, analysis has focused on examining the effect PA and/or strength have on OA between individuals (between-person comparison). Yet, how the variability in PA and strength over time within the same individual (within-person comparison) is associated with $O A$ is not well recognised. Statistical methods, such as multilevel models that properly capture the within-person processes can be used to tell us whether changes within an individual over time relate to changes in OA outcomes in that same individual.

Objectives: This study aimed to investigate the associations of between-person and within-person variability in PA and leg strength with knee cartilage volume loss over 10.7 years in older adults.

Methods: 479 community-dwelling older-adults (50\% female, mean age $61 \pm 6$ years, range $50-80$ years) were studied at baseline, $2.7,5.1$, and 10.7 years. PA (measured objectively as steps/day) and leg strength (measured objectively in $\mathrm{kg}$ ) were assessed at all four time-points. Knee cartilage volume was measured using MRI at baseline and 10.7 years. Linear mixed-effect regression models were used to estimate the association of between-person and within-person variability in PA and leg strength with cartilage volume loss over 10.7 years. Models were adjusted for age, sex, body mass index and history of knee injury or surgery.

Results: Mean cartilage volume loss over 10.7 years was $465 \pm 231 \mathrm{~mm}^{3}$. No between-person associations existed between PA and cartilage volume loss (Beta: 18.8 per 1000 steps/day, 95\% Cl -6.1, 43.7). However, within-person variability in PA was protectively associated with changes in cartilage volume, such that having higher PA compared to an individual's average level of PA minimised their cartilage volume loss over time (Beta: 32.8 per 1000 steps/day, 95\% Cl 20.8 44.6). Between-person effects showed that participants with greater leg strength lost less cartilage volume over time (Beta: 5.4 per $1 \mathrm{~kg}, 95 \% \mathrm{Cl} 3.1,7.8)$. Withinperson variability in leg strength was also protectively associated with changes in cartilage volume, such that having higher leg strength compared to an individual's average strength minimised their cartilage volume loss over time (Beta: 3.3 per $1 \mathrm{~kg}, 95 \% \mathrm{Cl} 2.1,4.5)$

Conclusions: Our unique analysis method adds a new perspective to the PA and OA debate. The implication of these findings demonstrate that individuals can minimise cartilage volume loss by increasing their own PA and strength, which supports the clinical recommendations of promoting PA and strength to prevent and treat $O A$

Disclosure of Interest: None declared

DOI: 10.1136/annrheumdis-2018-eular.3204

\section{FRI0542 POTENTIAL NOCICEPTIVE PAIN RELIEF OF INTRA- ARTICULAR SALINE CONTROL IN CLINICAL TRIALS OF KNEE OSTEOARTHRITIS: A SYSTEMATIC REVIEW AND META-ANALYSIS OF RANDOMISED TRIALS}

I. Simsek, T. Phalen, A. Bedenbaugh, J. Tambiah. Samumed, LLC, San Diego, USA

Background: Hyaluronic acid, corticosteroids and platelet-rich plasma (PRP) are widely used intra-articular $(\mathrm{IA})$ therapies for the management of mild to moderate knee osteoarthritis (OA). Many trials evaluating the efficacy of IA-administered therapies commonly use IA saline injections as a placebo comparator arm. A previously published systematic review Altman et al, 2016 showed significant reduc tions in pain relief with IA saline in both the short- (3 months) and long-term (6-12 months).

Objectives: The aim of this updated systematic review and meta-analysis was to assess the clinical benefit and harm associated with use of IA saline in trials of IA therapies for patients with painful knee OA

Methods: We searched MEDLINE and Embase databases for randomised controlled trials (RCTs) published up to and including October 12th, 2017. Two reviewers independently assessed the eligibility of potential reports and the risk of bias of included trials. We analysed short ( $\leq 3$ months) and long-term (6-12 months) pain reduction from baseline of the saline arm of included trials using standardised mean differences (SMDs; estimated assuming a null-effect in a comparator group) that were weighted and pooled using a random-effects model. Pain scores were transformed to a 100-point scale when necessary. We summarised and presented treatment-related adverse events (AEs) descriptively.

Results: We included 46 RCTs, of which 44 provided sufficient data to be included in the meta-analysis for benefit. IA saline significantly improved shortterm knee pain from baseline vs. a null effect for a comparator group across 36 studies involving 1908 patients (SMD $-0.85,95 \% \mathrm{Cl}-1.05$ to $-0.66 ; \mathrm{I}^{2}=87 \%$ ). There was also significant reduction in long-term knee pain following IA injection with saline across 25 studies involving 1758 patients (SMD $-0.78,95 \% \mathrm{Cl}-1.02$ to -0.55$)$ with a substantial degree of heterogeneity $\left(\mathrm{I}^{2}=90 \%\right)$. Thirty-three of the included trials reported on adverse events, none of which found any serious treatment-related AEs following IA injection with saline.

Conclusions: The pain relief observed with IA saline should prompt one to consider the added effectiveness of current IA treatments that use saline comparators in clinical studies, and challenges of classifying IA saline injection a "placebo." 


\section{REFERENCE:}

[1] Altman RD, et al. Semin Artrhritis Rheum 2016;46:151-9.

Disclosure of Interest: I. Simsek Shareholder of: Samumed, LLC, Employee of: Samumed, LLC, T. Phalen Shareholder of: Samumed, LLC, Employee of: Samumed, LLC, A. Bedenbaugh Shareholder of: Samumed, LLC, Employee of: Samumed, LLC, J. Tambiah Shareholder of: Samumed, LLC, Employee of: Samumed, LLC

DOI: 10.1136/annrheumdis-2018-eular.3823

\section{FRI0543 HEALTH RESOURCE USE AND COST-OF-ILLNESS OF SYMPTOMATIC KNEE AND/OR HIP OSTEOARTHRITIS : DATA FROM KHOALA COHORT}

J.H. Salmon ${ }^{1,2}$, A.C. Rat ${ }^{3,4}$, H. Achit ${ }^{4}$, W. Ngueyon Sime ${ }^{4}$, C. Gard ${ }^{5}$, F. Guillemin ${ }^{4,6}$, D. Jolly ${ }^{2,7}$, B. Fautre ${ }^{8,9}$. ${ }^{1}$ Rheumatology, Maison Blanche Hospital, Reims University Hospitals; ${ }^{2}$ University of Reims Champagne-Ardenne, Faculty of Medicine, EA 3797, Reims; ${ }^{3}$ Rheumatology, CHU de Nancy, Hôpitaux de Brabois; ${ }^{4}$ Université de lorraine, EA 4360 Apemac, Nancy; ${ }^{5}$ Pharmacy, Pitié-Salpêtrière Hospital, Paris; ${ }^{6}$ INSERM, CHRU Nancy, CIC 1433 Epidémiologie clinique, Nancy, ${ }^{7}$ Research and Innovation, Robert Debré Hospital, Reims; ${ }^{8}$ Rheumatology, Université Pierre et Marie Curie Curie; ${ }^{9}$ GRC08, Institut Pierre Louis de d'Epidémiologie et Santé Publique, Pitié-Salpêtrière Hospital, Paris, France

Background: Hip and knee Osteoarthritis are a major public health problem. Data on the economic impact are scarce.

Objectives: The purpose of our study was to estimate the annual direct costs of patients followed for hip and/or knee osteoarthritis from the KHOALA cohort.

Methods: The KHOALA cohort is a French population-based multicenter cohort of 878 patients with symptomatic knee and/or hip OA, aged between 40 and 75 years old recruited between 2007 and 2009. Direct costs were collected annually for 5 years. Costs were annualised and expressed in euros per patient.

Results: Over the 5 years, the average total direct costs were $€ 2575$ (sd 8085) per patient per year and median $€ 1015$ (IQR 430-2500) (figure 1).

Drugs represented the main health expenditure item ( $>50 \%$ of total costs). However, the proportion attributable to osteoarthritis drugs accounted for less than $5 \%$ of total costs. The second item of expenditure was hospitalizations (hip and/or knee arthroplasty represents $7 \%$ of total average costs). Medical consultations were the third item of health expenditure $(2.5 \%$ to $4 \%$ of total average costs). Physiotherapy represented $1 \%$ to $2 \%$ of total average costs.

Costs increased with older age, female sex, retirement, a high BMI, a high Charlson index and poor health status.

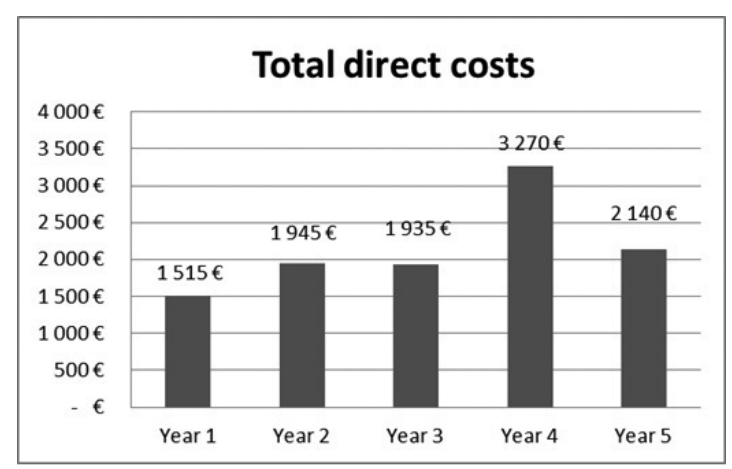

Abstract FRI0543 - Figure 1. Average annual total direct costs over 5 years per patient

Conclusions: These data are important results to describe the cost of care consumption of a sample of patients with symptomatic osteoarthritis of the hip and/or knee recruited to the general population in France. However, the specific cost attributable to osteoarthritis needs to be studied.

\section{REFERENCE:}

[1] Guillemin F, Rat AC, Mazieres B, Pouchot J, Fautrel B, Euller-Ziegler L, et al. Prevalence of symptomatic hip and knee osteoarthritis: a two-phase population-based survey. Osteoarthr Cartil OARS Osteoarthr Res Soc. Nov 2011;19(11):1314-22.

Disclosure of Interest: None declared

DOI: 10.1136/annrheumdis-2018-eular.4409
FRI0544

URINARY 6-SULFATOXYMELATONIN EXCRETION AND GALECTIN-3 PLASMA LEVEL IN PATIENTS WITH OSTEOARTHRITIS

K. Zaichko, O. Gumeniuk, M. Stanislavchuk. The Department of the Internal Medicine, National Pirogov Memorial Medical University, Vinnytsya, Ukraine

Background: Melatonin and galectin-3 are considered as factors in the development of immune-inflammatory and destructive changes in joints. ${ }^{1-5}$ Melatonin has chondrogenic and antinociceptive properties, ${ }^{2 ;} 4$ while galectin-3 plays an important role in cell-cell adhesion, macrophage activation, angiogenesis, and apoptosis. ${ }^{1 ; 3}$ The clinical and pathogenetic significance of melatonin and galectin-3 in osteoarthritis remains on the discussion.

Objectives: To study the excretion of 6-sulfatexymelatonin (metabolite of melatonin) and galectin-3 level in the blood and evaluate their association with the clinical manifestation and life quality in patients with $O A$.

Methods: Study involved 141 patients with OA of knee joints (76.6\% women), aged $58.4 \pm 7.91$ years, duration of the disease $10.5 \pm 6.50$ years $(M \pm S D) .47$ (33.3\%) patients had knee and hip OA, $38(27 \%)$ patients had reactive synovitis. The control group was presented by 36 practically healthy subjects $(72.2 \%$ female) aged $57.1 \pm 9.95$ years $(M \pm S D)$. 6-sulfatoxymelatonin (6-SMT) in urine and galectin- 3 in blood were determined by ELISA. The severity of pain, stiffness and physical functioning of the joints were evaluated by the Western Ontario and McMaster Universities Osteoarthritis Index (WOMAC). Quality of life was evaluated by Short Form-36 (SF 36).

Results: It was established in patients with OA a decrease in 6-SMT excretion, (mean 25.3 vs $38.8 \mathrm{ng} / \mathrm{mg}$ creatinine in control, $\mathrm{p}<0.001$ ). 6-SMT excretion correlated with age $(r=-0.40 ; p<0.001)$ and was more significant in patients with knee +hip OA (mean 26.5 vs $23.0 \mathrm{ng} / \mathrm{mg}$ creatinine in patients with OA of the knee only, $\mathrm{p}<0.001$ ). Lower levels of 6 -SMT excretion associated with higher pain and with lower quality of life. Patients with $O A$ had increased galectin-3 levels in the blood (mean 16.4 vs $10.1 \mathrm{ng} / \mathrm{ml}$ in the control, $\mathrm{p}<0.001$. In patients with $\mathrm{OA}$ of knee and hip joints were estimated higher levels of galectin-3. Levels of galectin-3 were significantly higher in patients with synovitis (mean $21.5 \mathrm{vs} .13 .8 \mathrm{ng} / \mathrm{ml}$ without synovitis, $\mathrm{p}<0.001)$. The increase of galectin- 3 in the blood was associated with a marked increase of the total WOMAC index and with decrease of life quality. The level of galectin-3 directly correlated with age, disease duration $(r=0.28$, $0.23, p<0.01)$ and inversely correlated with 6 -SMT excretion $(r=-0.28 ; p<0.01)$. Conclusions: Lower levels of melatonin and higher of galectin- 3 were associated with higher WOMAC index and poorer quality of life in patients with $\mathrm{OA}$. This association may reflect possible pathogenic role of melatonin and galectin-3 in OA.

\section{REFERENCES:}

[1] Forsman H, Islander U, Andréasson E, et al. Galectin 3 aggravates joint inflammation and destruction in antigen-induced arthritis. Arthritis Rheum 2011;63(2):445-454

[2] Hong $\mathrm{Y}$, Kim H, Lee $\mathrm{S}$, Jin $\mathrm{Y}$, et al. Role of melatonin combined with exercise as a switch-like regulator for circadian behavior in advanced osteoarthritic knee. Oncotarget 2017;8(57):97633-97647.

[3] Hu Y, Yéléhé-Okouma M, Ea HK, et al. Galectin-3: A key player in arthritis. Joint Bone Spine 2017;84(1):15-20.

[4] Pei M, He F, Wei L, Rawson A. Melatonin enhances cartilage matrix synthesis by porcine articular chondrocytes J. Pineal. Res. 2009;46(2):181187.

Disclosure of Interest: None declared

DOI: 10.1136/annrheumdis-2018-eular.3778

\section{FRI0545 RISK FACTORS PREDICTING RADIOLOGICAL PROGRESSION OF KNEE OSTEOARTHRITIS}

L. Alekseeva ${ }^{1}$, S. Anikin ${ }^{1}$, N. Kashevarova ${ }^{1}$, E. Sharapova ${ }^{1}$, E. Taskina ${ }^{1}$ T. Korotkova ${ }^{1}$, T. Raskina ${ }^{2}$, E. Otteva ${ }^{3}$, A. Dubikov ${ }^{4}$, E. Zonova ${ }^{5}$, I. Vinogradova ${ }^{6}$ ${ }^{1}$ FSBI V.A. Nasonova Research Institute of Rheumatology, Moscow, ${ }^{2}$ 2. FSBEI HE KemSMU MOH, Kemerovo; ${ }^{3}$ 3. Regional SBHI CDC MOH Khabarovsky kray, Khabarovsk; ${ }^{4}$ Pacific State Medical University, Vladivostok; ${ }^{5} 5$. FSBEI HE Novosibirsk State Medical University, MOH, Novosibirsk; ${ }^{6} 6$. SHI Ulyanovsk regional affiliated hospital, Ulyanovsk, Russian Federation

Background: Currently a number of risk factors (RF) are considered to be responsible for radiological progression of knee osteoarthritis (OA), nevertheless key predictors of OA progression have not yet been established.

Objectives: To identify RF predicting radiological progression of knee joint osteoarthritis $(\mathrm{OA})$ in a 5 year multicenter prospective study.

Methods: This study of RF predicting knee OA progression was the first with multicenter prospective design ever conducted in Russia. The study included 344 female patients 40-75 y.o with primary stage I-III knee OA (ACR criteria) from 6 centres. Radiological stage was identified by Kellgren J.- Lawrence J. grading 